\title{
MODEL MANUSCRIPT
}

\section{Title: Genus species sp. nov., an undescribed fungus (Taxonomic group) from habit in country with interesting biological properties}

\begin{abstract}
If your title is sufficiently engaging, a prospective reader will probably look next at the Abstract. The abstract should include all details necessary for the reader who does not have access to the whole article (i.e. someone looking at the abstract only on-line or in an abstract journal) so that they will know whether it is worth their time or money to obtain the full article. When describing a new species, you should include a summary of the diagnostic characters of the new species, especially the spore characters and dimensions. Make sure to include information about where your fungus was found and what it was growing on. If you have molecular data, it is useful to mention what genes you have sequenced, and what this information tells us about the fungus, such as what family or order it belongs to, and what are the most closely related species. Mention if a key to related species or comparative synoptic table is included, a feature that will increase potential readership.
\end{abstract}

\begin{abstract}
Key words:
These should not reproduce words in the title. It is useful to list special techniques used in the description, e.g. electron microscopy, DNA sequencing, or chemotaxonomic methods.
\end{abstract}

Article info: Submitted: dd month yyyy; Accepted: dd month yyyy; Published: dd month yyyy.

\section{INTRODUCTION}

The paragraphs of the introduction should be presented in a logical order, i.e. how they tell the best story. Remember, most people reading a scientific paper will only read the Introduction and Discussion, so the account of your discovery should be complete and complementary between these two sections. Normally, you will tell the reader about the larger projects (if any) that led to the discovery of the new species, provide information on its ecological niche and associated organisms, and give references to complementary publications where appropriate. Information should be provided about the taxonomy of genus in which the species is being described, such as the number of species already known, a brief review of recent revisions or monographs, perhaps discussion of controversies about the generic concept, and something about the biology of the species. Be diligent about citing all relevant literature.

If you have DNA sequence data, usually one paragraph will provide a brief review of the existing state of molecular knowledge for the group your species belongs to, and explain to the reader what experiments you have done with your own species to fit it into the existing context.

Another paragraph gives information about why the new species is suspected to be undescribed. This should be basic information that leads into the formal part of the paper. Some of this information may be repeated and presented in more detail in the discussion.

Some papers may require a longer introduction. Manuscripts including molecular or physiological data are often longer. Situations where the new fungus could be described in one of several different genera also may require a longer introduction. Sometimes this will include a more extensive review of historical literature, or discussions of taxonomic characters of particular significance. You must judge whether this information is most suitable in the introduction, which the reader will read before the description itself, or if it is more logical to place it in the discussion.

Usually the Introduction concludes with a statement like, "therefore, we decided that our fungus represents an undescribed species, which is described and illustrated here as Genus species sp. nov."

\section{MATERIALS AND METHODS}

This section is often omitted from taxonomic papers that include only morphological data, but it is preferable to include as many details as possible. Some of the following subheadings and paragraphs might be appropriate.

\section{Collecting and field sites}

How the specimens were collected and transported to the laboratory and preserved or incubated prior to examination may be relevant. Information about specific field sites is usually given in the 'Material examined' section, but it might be appropriate to provide more details here if they are relevant to understanding the species.

\section{Cultures and media}

Give recipes for the isolation media employed, or cite a reference for the media. Give brand names for extracts used, such as malt extract, yeast extract, and the agar used for the media. Describe the inoculation methods, incubation conditions such as temperature and lighting 
regime, and length of incubation before examination. Determine the cardinal growth temperatures (minimum, optimum and maximum) if possible. List the culture collections where the cultures are maintained, with accession numbers, either here or in a table.

\section{Isolation methods}

Explain all isolation methods used, such as explants from sporophores or infected host tissues, removal of spores directly from sporulating structures, transfer of actively discharged spores from Petri dish lids or spore prints, etc. If the substrate was treated before isolation, e.g. by some form of surface sterilization, these methods should be explained. Give the recipes for the isolation media employed including any antibacterial compound added, or cite a reference for those media. Describe the incubation conditions such as temperature and lighting conditions. If singlespore cultures were prepared, a good practice to be undertaken when possible (Choi 1999, Crous 2002), describe how this was done.

\section{Microscopy}

Give details of the kind of microscope used, including the illumination systems (e.g. phase contrast, differential interference contrast), the mounting media and stains employed for routine examination and for making measurements, and how many structures of each microscopic character were measured. A similar section, with details on dehydration protocols, fixation, staining, etc. should be given for electron microscopy methods, if these were used. Permanent microscopic preparations should be deposited with the type if possible, which will make your observations reproducible to later taxonomists, and limit the amount of material they might use on specimens in future studies.

Techniques used for illustrations are often given here, but may also be briefly mentioned in the figure legends. For example, whether drawings were made with a drawing tube, a camera lucida, or by freehand, and the type of camera used for photography, may be relevant. It is essential to describe techniques used for image enhancement of digital photographs, such as the sharpening filters of PhotoShop or other imaging software, whether separate photographs were combined into one image, or whether colours have been altered (Microscopy Society of America, 2003). Modification of contrast has always been standard practice in photography and need not be mentioned.

\section{Physiological tests or chemotaxonomic methods}

For yeasts, substrate utilization and other physiological tests are standard parts of taxonomic descriptions. For some lichen groups, spot-tests with a standard set of chemical reagents are essential. These methods must be described carefully. If chemotaxonomic methods, such as isozyme analysis or secondary metabolite profiling, were employed, complete methods should be given to allow the resulting data to be reproduced.

DNA extraction, PCR amplification, DNA sequencing, and phylogenetic analysis Note what kind of material was used for DNA extraction, such as cultures, single spores, or naturally occurring tissues, and give the details of the kits and methods used for DNA extraction. For DNA isolations from natural tissues, note whether procedures were repeated to reduce the chance of sequencing a contaminant or associated organism. Give the details of PCR amplification profile used, the concentrations of reagents used in the reactions, and information on the brand and model of thermocycler used. Provide details of any methods used to clean or otherwise process the PCR products before sequencing. If $P C R$ products or other DNA fragments were cloned for sequencing, provide the relevant information for this procedure. For the DNA sequencing, provide details of the cycle sequencing profile used, the concentration of reagents used, the brand and model of the thermocycler, the relevant information about the sequencing chemistry used, and the brand and model of DNA sequencer employed. If you used a DNA sequencing service, list it here. Cite the literature where PCR and sequencing primers were first published. If you designed the primers yourself, give details of how you did this. Include details about how you did your phylogenetic analyses, including literature citations for sequences originating from published or unpublished work of colleagues, the software used for analysis, and the details of the parameters used for the analysis. Diverse methods of phylogenetic analysis are available. While the choice of methods is largely a matter of preference, there is a general agreement that it is critical to employ measures of confidence, such as the bootstrap, Bayesian posterior probabilities, the 'decay index' or congruence among independent data sets. If you have used several sequences from a previously published study, you should cite that study so that it is properly credited (Seifert et al. 2008).

\section{RESULTS}

Many descriptions of new species will not have a Results section because all of the data are included in the Taxonomy section. However, if some experiments were done with the fungus, such as physiological tests or tests of antibiotic resistance, then these data should be presented in the Results section, in the same order as the methods are described in the Materials and Methods section. Often, the results of physiological tests are given in a Table. 
General results of DNA sequencing analyses are usually given in this section. These can include details of the length and composition of the DNA fragments, and the results of comparisons with other sequences (e.g. BLAST searches). If phylogenetic trees are presented, the tree statistics may be given in this section, or otherwise in the figure legends. Describe what the data shows, e.g. that the sequence is similar to those in a particular genus, family or order, or that the sequence is apparently unique, but leave the conclusions derived from this for the Discussion section. Mention support values for the critical nodes in your tree based on bootstrap frequencies or other measures of confidence. If you have done analyses using different phylogenetic methods, or have analyzed several genes, then comparison of the results is appropriate here, but leave the conclusions for the Discussion section.

\section{TAXONOMY}

Genus species Authors ${ }^{1}$, sp. nov. MycoBank: MBxxxxxxx.

Figs $x x x-x x x$

The Latin diagnosis comes first and is essential for valid publication. It should list the diagnostic characters only, i.e. those that separate it from similar ones, and not be a complete translation of the description. Many journals now restrict the Latin to a few lines. Use published Latin diagnoses for models, then if possible have yours checked by a mycologist or botanist competent in scientific Latin. If there is no such expert in your own department, consult with colleagues in other institutions. Stearn's Botanical Latin (1992) is a valuable resource for preparing Latin diagnoses.

Holotypus: Collection acronym, accession number. Immediately after the Latin diagnosis, clearly and explicitly indicate the details of the single accession that will serve as holotype. If you wish to list isotypes or extype cultures here, be certain that they are clearly distinguished from the holotype or you may have problems with the validity of your new name (Art. 37.7).

A full description follows. Think of the descriptions and illustrations together as providing a blue-print for your new species. If someone wanted to build an exact scalemodel of the fungus, they should be able to do so using your paper. Where both asexual and sexual states (i.e. anamorph and teleomorph) occur, the description of the sexual state is traditionally given first. In general, a taxonomic description begins at the broadest scale

${ }^{1}$ Names of author or authors, and their abbreviations, should follow the standards of Kirk \& Ansell (1992). and moves towards the finest. Macroscopic characters are described next. Use a colour standard, such as Ridgway (1912), Rayner (1970), Kornerup \& Wancsher (1984), or Munsell (1905 and many subsequent editions) to accurately describe colours. Most details will be visual, but sometimes texture and odour are useful additions. When describing microscopic characters, be as complete as possible about shape, colour, texture and size for every component of the fungus. There are standard terminologies for shape; check Ainsworth \& Bisby's Dictionary of the Fungi (Kirk et al. 2008, and earlier editions, see under 'shapes') as a starting point. Be aware that the terminology for describing three dimensional shapes sometimes differs from the terminology used to describe two dimensional shapes. The full range of observed dimensions should be given for all structures. Means should be calculated for all dimensions in the description (at least the spores), along with a statistical measure of variation in these measurements, such as standard error, standard deviation, confidence intervals or percentile ranges.

If you have isolated a culture, its features are usually included in a separate paragraph. For some fungi, colony characters are described first; for others, this information follows the morphological description. At a minimum, give the growth rates on a specified medium and explain the temperature and light regime, and give a general impression of the colour and texture of the colonies. If the fungus sporulates in culture, it can be very helpful to compare the sizes and shapes of the microscopic structures to what occurred on the natural specimen. The detail employed in culture descriptions varies considerably from one taxonomic group to another, and you should consult published descriptions for the group you are working with.

If you have done any physiological tests, or determined cardinal temperatures, this information is normally put in a separate paragraph in the description. It can also be put into a table or in a graph, which may be easier for a reader to follow.

Substrate or Host: Provide a summary of the known hosts or substrates as a separate paragraph, especially if you have more than one specimen.

Distribution: Summarize the known disttibution, by continent, country (and by province or state for larger countries), along with relevant information on the biome, climactic or geological conditions.

Etymology: Explain the meaning or derivation of the species epithet, and note the language of origin of the word(s) used for constructing the epithet. Avoid species epithets with more than five syllables and those similar to other epithets in the same genus. Epithets that are descriptive are most helpful, but names can be derived from any source, including acronyms or the name of a 

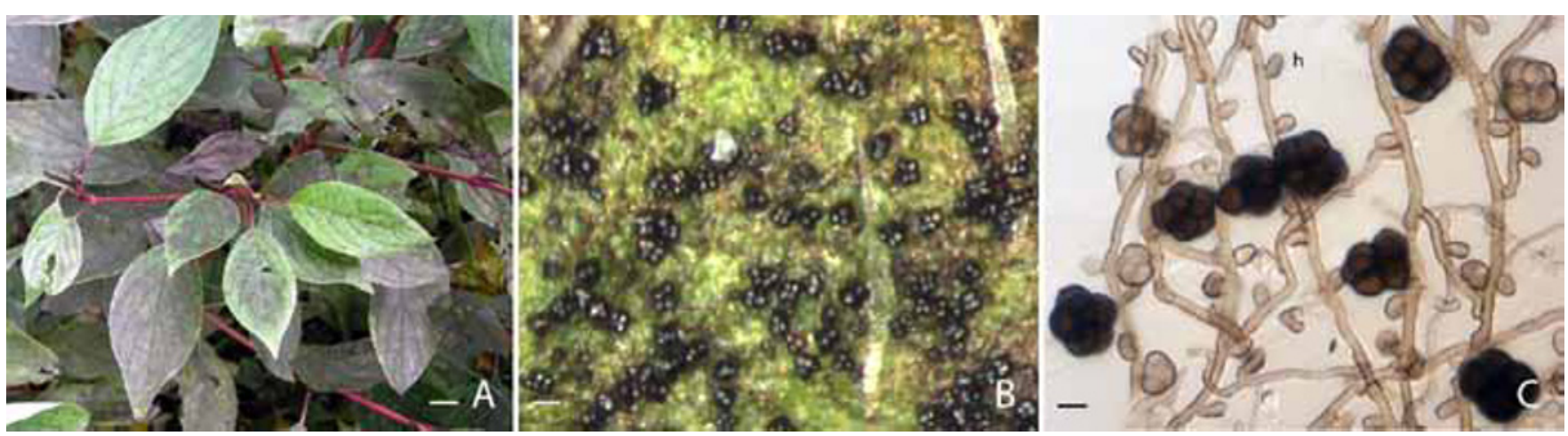

Fig. 1. Genus species (specimen or culture number, noting whether it is type). A series of photographs of the fungus, showing the field habit, the appearance under the dissecting microscope, and microscopic photographs showing taxonomically relevant structures and preferably some developmental sequence. All illustrations should include scale bars. In this particular example:

Fig. 1. Sarcinella questierii (DAOM 235813). A. Black growth on living leaves of Cornus sp. B. Black conidia on leaf surface. C. Development of dictyoconidia from conidiogenous hyphae, with hyphopodia $(\mathrm{h})$ arising from the same hyphae (differential interference contrast). Bars: $A=1 \mathrm{~cm}$, $B=25 \mu \mathrm{m}, C=10 \mu \mathrm{m}$. B-C, composite images created with CombineZ (Hadley 2006).

person, usually someone involved in the discovery of the fungus or a mycologist who has made a significant contribution to the subject.

Additional material examined: Most journals have a specific format for this part of the paper. For all specimens and cultures, including isotypes and extype cultures, list Country: Province/State/Territory/ County/Township, City/Town/Park, Specific location details (GPS coordinates, including altitude), Substrate or host, Date of collection and/or isolation, Collector's name, Collector's number (if any), Herbarium or culture collection abbreviations and accession numbers where the material is preserved. Often, some of this information is instead provided in a table including GenBank accession numbers for DNA sequences.

\section{DISCUSSION}

The discussion completes the story that began in the Introduction. There are many ways to write this section, but one rule is not to introduce new data that should have been introduced in the 'Results' or 'Taxonomy' sections. Mixing of the Results and Discussion in one section is generally frustrating for the reader, unless the section is very short.

It is often useful to start the Discussion by summarizing the diagnostic features of the fungus you have described.

In a separate paragraph, you should compare your fungus to other similar species of the same genus, stating clearly how they differ. Many papers will include either a diagnostic key or synoptic table (or both), either including all species of a smaller genus, or only the most similar species of a larger genus, to assist the reader in understanding why the new species is distinct.
If you have DNA sequence data, there are often several paragraphs of discussion relating to what they show or do not show. Discuss how the data support the classification of your fungus and its recognition as a distinct species. If you have done analyses using different phylogenetic methods, or have analyzed several genes, compare the results and explain your conclusions carefully, especially if contradictory evidence occurs in the different data sets or analyses.

It is often useful to conclude the paper with discussion of the biology of the new species, either demonstrated by the experiments done in the paper or as an extension of field observations. A limited amount of speculation on this topic is usually tolerated by reviewers and editors.

\section{ILLUSTRATIONS}

The ICBN does not require that new species descriptions have illustrations (Fig. 1), but few journals allow the description of a new species without them, with the exception of yeasts. Usually, at least some of the illustrations will be of the holotype specimen or culture. The package of illustrations should present the complete concept of the species to the reader so that they can confidently identify your fungus. Provide visual information at several different size scales, from general habitat to the most detailed microscopy. Expectations vary among different taxonomic groups, but often a mixture of photographs and line drawings are included. Individual photographs are visual data that are proof of observations. Electron micrographs are generally unhelpful to facilitate identification, but scanning electron micrographs may be necessary for documenting spore ornamentation or tissue types, and transmission electron micrographs may be necessary to prove ultrastructural observations 
of spore production (especially types of conidiogenesis). Line drawings are interpretations; they are not proof, but can present a complex concept in one image and be extremely helpful for someone trying to identify your species. With colour photographs now published with increasing frequency, and the relative economy and ease of digital photography and computerized imaging, the preparation of informative illustrations is one of the most exciting aspects of describing a new species. Find the best model illustrations for the group of organisms where your species fits, and then do better!

\section{ACKNOWLEDGEMENTS}

In addition to the usual acknowledgements for a scientific paper (e.g. mentors, sources of funding), it is traditional to acknowledge any taxonomic specialists that you have consulted during your decision to describe a new species. Similarly, it is customary to acknowledge the curators of any collections who have provided specimens or cultures that was used in your study. For this paper, we wish to thank the other members of the International Commission on the Taxonomy of Fungi, especially David Hibbett and David Hawksworth, and the peer reviewers, for valuable comments on earlier drafts of this paper. The final sentence usually identifies funders of the research.

\section{REFERENCES}

Do your best to cite the relevant historical and modern literature, including revisions, monographs, identification keys, and molecular studies that you have consulted. Reviewers often see submitted papers in some fields of taxonomy that only cite literature more than 25 years old. Study the guidelines of the journal carefully for citation formats.

Cantino PD, Queiroz K de (2010) International Code of Phylogenetic Nomenclature. Version 4c. <ohio.edu/ phylocode/>

Choi Y-W, Hyde KD, Ho WH (1999) Single spore isolation of fungi. Fungal Diversity 3: 29-38.

Constantinescu O (1983) Dried reference fungal cultures. A review and a simpler technique. Bulletin of the British Mycological Society 17: 139-143.

Crous PW (2002) Adhering to good cultural practice (GCP). Mycological Research 106: 1377-1378.

Crous PW, Gams W, Stalpers JA, Robert V, Stegehuis G (2004) MycoBank: an online initiative to launch mycology into the 21st century. Studies in Mycology 50: 19-22.

Hadley A (2006) CombineZ. Version 5. Published by the author. <hadleyweb.pwp>

Hawksworth DL (1974) Mycologist's Handbook: an introduction to the principles of taxonomy and nomenclature in the fungi and lichens. Kew: Commonwealth Mycological Institute.
Holmgren, PK, Holmgren NH, Barnett LC (1990) Index Herbariorum. Part I. The Herbaria of the World. $8^{\text {th }}$ edn. [Regnum Vegetabile vol. 120.] Utrecht: Bohn, Scheltema \& Holkema. <sweetgum.nybg.org/ih/>

Kirk PM, Ansell AE (1992) Authors of Fungal Names: A list of authors of scientific names of fungi, with recommended standard forms of their names, including abbreviations. [Index of Fungi Supplement.] Wallingford, UK: CAB International. <indexfungorum.org/Names/ AuthorsOfFungalNames.asp>

Kirk PM, Cannon PF, Minter DW, Stalpers JA, eds (2008) Ainsworth and Bisby's Dictionary of the Fungi. $10^{\text {th }}$ edn. Wallingford, UK: CAB International.

Kohlmeyer J, Kohlmeyer E (1972) Permanent microscopic mounts. Mycologia 64: 666-669.

Kornerup A, Wanscher JH (1984) Methuen Handbook of Color. $3^{\text {rd }}$ edn. London, UK: Methuen.

Lapage SP, Sneath PHA, Lessel EF, Skerman VDB, Seeliger HPR, Clark WA (eds) (1992) International Code of Nomenclature of Bacteria and Satutes of the International Committee on Systematic Bacteriology and Statutes of the Bacteriology and Applied Microbiology Section of the International Union of Microbiological Societies. Bacteriological Code (1990 Revision). Washington, DC, USA: American Society for Microbiology.

McNeill J, Barrie FR, Burdet HM, Demoulin V, Hawksworth DL, Marhold K, Nicolson DH, Prado J, Silva PC, Skog JE, Wiersema JH, Turland NJ, eds (2006) International Code of Botanical Nomenclature (Vienna Code). [Regnum Vegetabile vol. 146.] Liechtenstein: Gantner Verlag, Ruggell. <ibot.sav.sk/icbn/main.htm>

Microscopy Society of America (2003) MSA policy on digital imaging. www.microscopy.org/resources/digital_imaging. cfm

Munsell AH (1905) A Color Notation. Boston: G. H. Ellis. $<$ triplecode.com/munsell/> (for a pop-up computer version).

Rayner RW (1970) A Mycological Colour Chart. Kew: Commonwealth Mycological Institute.

Ridgway R (1912) Color Standards and Color Nomenclature. Washington, DC, USA: published by the author. </hldigital. lindahall.org/u?/nat_hist,1444>

Seifert KA, Crous PW, Frisvad JC (2008) ACT: Appropriate Citation of Taxonomy. Inoculum 59(3): 4; Persoonia 20: 105.

Sigler L, Hawksworth DL (1987) International Commission on the Taxonomy of Fungi (ICTF): Code of practice for systematic mycologists. Mycologist 1: 101-105; Mycopathologia 99: 3-7; Microbiological Sciences 4: 83-86.

Stearn WT (1992) Botanical Latin: history, grammar, syntax, terminology and vocabulary. $4^{\text {th }}$ edn. Newton Abbot: David \& Charles.

Winston JE (1999) Describing Species: practical taxonomic procedure for biologists. New York: Columbia University Press.

World Federation for Culture Collections. s.d. <wdcm.nig ac.jp/wfcc/index.html> 